\title{
ADOLESCENTES COM SONOLÊNCIA DIURNA EXCESSIVA PASSAM MAIS TEMPO EM COMPORTAMENTO SEDENTÁRIO
}

\author{
ADOLESCENTS WITH EXCESSIVE DAYTIME SLEEPINESS SPEND MORE TIME \\ IN SEDENTARY BEHAVIOR
}

\author{
LOS ADOLESCENTES CON SOMNOLENCIA DIURNA EXCESIVA PASAN MÁS TIEMPO \\ EN COMPORTAMIENTO SEDENTARIO
}

\author{
Érico Pereira Gomes Felden \\ (Educador Físico) \\ Douglas Filipin' \\ (Educador Físico) \\ Diego Grasel Barbosa' \\ (Educador Físico) \\ Rubian Diego Andrade ${ }^{1}$ \\ (Educador Físico) \\ Carolina Meyer' \\ (Educadora Física) \\ Thaís Silva Beltrame \\ (Educadora Especial) \\ Andreia Pelegrini \\ (Educadora Física)
}

1. Universidade do Estado de Santa Catarina (UDESC), Florianópolis, SC, Brasil.

\section{Correspondência:}

Universidade do Estado de Santa Catarina. Centro de Ciências da

Saúde e do Esporte.

Rua Paschoal Simone, 358.

Coqueiros, Florianópolis, Brasil. 88080-350. ericofelden@gmail.com

\section{RESUMO}

Introdução: A diminuição da atividade física, a baixa duração do sono e o aumento do tempo sentado têm sido cada vez mais percebidos na adolescência. Objetivos: Investigar a associação entre comportamento sedentário e variáveis de sono (sonolência diurna e duração de sono) de adolescentes de um município de pequeno porte do sul do Brasil. Métodos: Trata-se de uma amostra representativa, de base escolar, do município de Maravilha, SC, Brasil, formada por 516 adolescentes de ambos os sexos, com 10 a 19 anos de idade. Foram investigadas as associações entre comportamentos sedentários, sono, percepção de estresse e atividade física. O comportamento sedentário foi investigado por meio do tempo sentado durante o dia. Além disso, outras questões foram investigadas, como: sexo, faixa etária, turno escolar, local de domicílio, renda e grau de instrução do chefe de família, duração do sono e sonolência diurna, percepção de estresse e nível de atividade física. Resultados: A prevalência de baixa duração do sono foi de 53,6\%. A média de tempo sentado durante um dia de semana foi de 382,2 min., sendo superior no sexo feminino $(p=0,001)$. Os adolescentes com baixa duração do sono apresentaram valores médios mais altos de tempo sentado $(p<0,001)$, sonolência diurna ( $p<0,001)$ e sonolência em sala de aula $(p<0,001)$. Os adolescentes com maior sonolência tinham 4,97 (IC: 2,50-9,87) vezes mais chance de ter comportamento sedentário elevado. Conclusão: Adolescentes com maior sonolência diurna apresentaram maior tempo dedicado aos comportamentos sedentários. Assim, as medidas de educação para um estilo de vida mais ativo na adolescência devem observar também questões gerais sobre sono e sonolência diurna.

Descritores: sono; estilo de vida sedentário; adolescente; distúrbios do sono por sonolência excessiva.

\section{ABSTRACT}

Introduction: Decreased physical activity, short sleep duration, and increased time sitting have been increasingly perceived in adolescence. Objectives: To investigate the association between sedentary behavior and sleep variables (daytime sleepiness and sleep duration) of adolescents in a small city in the south of Brazil. Methods: This is a representative school-based sample in the city of Maravilha, SC, Brazil, composed of 516 adolescents of both sexes, from 10 to 19 years old. Associations were investigated among sedentary behaviors, sleep, perception of stress and physical activity. Sedentary behavior was investigated by mean of sitting time during the day. In addition, other issues were investigated as sex, age, school shift, place of residence, income, and level of education of household head, sleep duration, and daytime sleepiness, perception of stress and level of physical activity. Results: The prevalence of short sleep duration was 53.6\%. The average time sitting during a weekday was $382.2 \mathrm{~min}$, being higher in females $(p=0.001)$. Adolescents with short sleep duration had higher average values of sitting time ( $p<0.001)$, daytime sleepiness $(p<0.001)$ and sleepiness in classroom ( $p<0.001$ ). Adolescents with highest sleepiness were 4.97 (Cl: 2.50 to 9.87) times more likely to have high sedentary behavior. Conclusion: Adolescents with higher daytime sleepiness showed greater time spent on sedentary behaviors. Thus, the education measures for a more active lifestyle during adolescence should also observe general questions about sleep and daytime sleepiness.

Keywords: sleep; sedentary lifestyle; adolescent; disorders of excessive somnolence.

\section{RESUMEN}

Introducción: La disminución de la actividad fisica, la baja duración del sueño y el aumento del tiempo sentado se han percibido cada vez más en la adolescencia. Objetivos: Investigar la asociación entre el comportamiento sedentario y las variables del sueño (somnolencia diurna y la duración del sueño) de adolescentes de una pequeña ciudad en el sur de Brasil. Métodos: Se trata de una muestra representativa basada en la escuela en la ciudad de Maravilha, SC, Brasil, compuesta de 516 adolescentes de ambos sexos, con 10 a 19 años de edad. Se investigaron las asociaciones entre los comportamientos sedentarios, el sueño, percepción de estrés y la actividad física. El sedentarismo fue investigado por medio de tiempo sentado durante el día. Además, otros temas se investigaron como el sexo, la edad, la jornada escolar, el lugar de residencia, ingresos y nivel de educación del jefe de hogar, la duración del sueño y la somnolencia diurna, la 
percepción del estrés y el nivel de actividad física. Resultados: La prevalencia de la corta duración del sueño fue 53,6\%. El promedio de tiempo sentado durante un día de semana fue 382,2 min., siendo mayor en las mujeres $(p=0,001)$. Los adolescentes con baja duración del sueño tenían valores medios más altos de tiempo sentado $(p<0,001)$, somnolencia diurna $(p<0,001)$ y la somnolencia en la clase $(p<0,001)$. Los adolescentes con mayor somnolencia diurna presentaron 4,97 (IC:2,50-9,87) veces más probabilidades de tener alto comportamiento sedentario. Conclusión: Los adolescentes con mayor somnolencia diurna mostraron mayor tiempo dedicado al comportamiento sedentario. Por lo tanto, las medidas de educación para un estilo de vida más activo durante la adolescencia también deben observar cuestiones generales sobre el sueño y somnolencia diurna.

Descriptores: sueño; estilo de vida sedentario; adolescente; trastornos de somnolencia excesiva.

\section{INTRODUÇÃO}

A expansão na criação de máquinas e equipamentos advinda da revolução industrial permitiu ao ser humano ter mais conforto e comodidade. Desta forma, são perceptíveis as mudanças nos comportamentos, como a diminuição das atividades físicas e o aumento do tempo sentado'. Adolescentes são especialmente afetados por este processo e apresentam acentuada diminuição de atividade física diária e aumento do comportamento sedentário ${ }^{2,3}$. Assim, embora a atividade física e o comportamento sedentário são considerados variáveis independentes, estas podem estar associadas com efeitos semelhantes em relação à saúde humana ${ }^{4}$.

Outra variável fundamental para a saúde, o sono, também sofreu modificações importantes nas últimas décadas. O sono inadequado tem sido associado a uma série de distúrbios comportamentais e de saúde em jovens ${ }^{5}$. Além disso, a redução do tempo total de sono pode estar associada ao dormir mais tarde e não ao acordar mais cedo ${ }^{6}$, por isso, as atividades antes de dormir podem implicar no atraso do sono. Essas atividades incluem o uso de mídia em tela eletrônica e outras atividades não relacionadas ao uso de telas, como fazer a lição de casa e exercícios aeróbios vigorosos.

A redução do tempo total do sono dos adolescentes, em consonância às obrigações com horários escolares, culmina no aumento dos níveis de sonolência diurna excessiva ${ }^{7}$. As demandas curriculares, assim como os horários ocupados para os estudos, parecem desregular o padrão do sono, pois os alunos sincronizam o ciclo vigília-sono diferenciadamente do ciclo claro-escuro para tentar cumprir suas atividades, acarretando problemas com a regularidade do sono ${ }^{8}$.

De forma geral, as prevalências de sonolência diurna e baixa duração do sono têm aumentado com o passar dos anos ${ }^{9}$, e, a atividade física e o comportamento sedentário são potenciais fatores associados às mudanças da expressão do ciclo vigília-sono ${ }^{10}$. No entanto, poucas evidências científicas estão disponíveis, especialmente em adolescentes, considerando as associações entre os comportamentos sedentários, atividade física e sono. Ainda, destaca-se que a literatura especializada é bastante restrita, considerando os hábitos de atividade física, comportamento sedentário e sono em cidades de pequeno porte, sendo mais comuns estudos conduzidos em grandes cidades. Assim, o presente estudo teve como objetivo investigar a associação entre comportamento sedentário e variáveis de sono de adolescentes do município de Maravilha, SC, Brasil.

\section{MATERIAIS E MÉTODOS}

A população do estudo consistiu de 2969 escolares, sendo 1757 na faixa etária de 10 a 14 anos e 1212 na faixa etária de 15 a 19 anos ${ }^{11}$ (IBGE, 2010). Participaram da amostra 516 adolescentes (263 rapazes), com média de idade de 14,57(1,77) anos do município de Maravilha, SC, Brasil. Trata-se de uma amostra representativa dos adolescentes do município de base escolar. O tamanho da amostra foi definido utilizando a proposta de Luiz e Magnanini ${ }^{12}$ considerando um erro amostral de cinco pontos percentuais e efeito de delineamento de 1,5. A proporção necessária para compensar eventuais perdas foi de 40\%. Após o cálculo amostral, foi considerado aceitável um número amostral mínimo por conglomerado de 437 sujeitos. A seleção da amostra foi por conglomerado e estratificada por faixa etária: na 1a faixa etária estavam os adolescentes de 10 a 14 anos e na $2^{\text {a }}$, adolescentes com idades de 15 a 19 anos. Para garantir a representatividade da amostra foi realizado levantamento das escolas estaduais que tinham alunos na faixa etária de análise. Foram localizadas seis escolas que recebem alunos das diversas regiões do município. Destas, cinco consentiram a realização da pesquisa. A partir deste consentimento foram sorteadas turmas de forma proporcional a escola e faixa etária com vistas ao atendimento do número mínimo necessário de adolescentes para a análise.

Os adolescentes responderam a um questionário estruturado contendo questões sobre os seguintes fatores: a) comportamento sedentário representado pelo tempo sentado; b) fatores sociodemográficos: sexo, idade, local de domicílio (rural ou urbano), turno de estudo (diurno ou noturno), renda mensal (salários mínimos brasileiros recebidos pela família) e grau de instrução do chefe de família (anos de estudo); c) fatores relacionados ao sono: duração do sono e sonolência diurna; d) percepção de estresse; e e) atividade física.

O comportamento sedentário foi verificado por meio do tempo sentado, o qual foi investigado pela pergunta "Quanto tempo no total você gasta sentado durante um dia de semana? ${ }^{13}$. Esta questão foi extraída do Questionário Internacional de Atividade Física e considera em sua análise o tempo em que o adolescente permanece sentado todo dia. Esta análise não inclui o tempo gasto sentado durante o transporte em ônibus, trem, metrô ou carro. O tempo sentado foi considerado a variável dependente deste estudo, e pelo fato deste não apresentar um ponto de corte amplamente aceito pela literatura, optou-se por categorizá-lo em tercis. Assim classificou-se como "pouco tempo sentado" (primeiro tercil = zero a $300 \mathrm{~min}$ ); tempo sentado moderado (segundo tercil = 301 a 479 min) e muito tempo sentado (terceiro tercil = acima de $480 \mathrm{~min}$ ).

A duração do sono foi analisada pelo tempo na cama considerando os horários de dormir e acordar nos dias com aula. Os adolescentes foram classificados com baixa duração de sono quando essa era inferior a 8 h por dia ${ }^{(8)}$. A latência do sono foi investigada por meio da questão "Você sente dificuldades para pegar no sono?", podendo o adolescente responder as alternativas: a) "nunca", b) "às vezes", ou c) "sempre" 8,14.

A sonolência diurna foi investigada pela Pediatric Daytime Sleepiness Scale (PDSS) desenvolvida para avaliar a sonolência diurna de crianças e adolescentes ${ }^{15}$ e validada para o Brasil por Felden et al. ${ }^{16}$. Esta escala é formada por oito questões com respostas organizadas em uma escala Likert de quatro pontos de "nunca" a "sempre". A pontuação da escala varia de zero a 32 pontos. Seus escores são analisados de forma contínua sendo que o valor mais alto representa mais sonolência diurna. Da mesma forma, como na análise do comportamento sedentário, a PDSS, por não apresentar um ponto de corte amplamente aceito, foi analisada pelos tercis na análise multinomial: primeiro tercil = zero a 12 pontos; segundo tercil = 13 a 18 pontos; e terceiro tercil acima de 18 pontos.

A sonolência em sala de aula foi analisada por meio da primeira 
questão da PDSS que se refere, especificamente, a esta situação. Neste caso, os adolescentes foram classificados como tendo sonolência em sala de aula "sempre", "às vezes" ou "nunca".

Por fim, os adolescentes foram questionados quanto ao seu nível de atividade física por meio do questionário proposto por Florindo et al. ${ }^{17}$. Este instrumento é composto por 17 questões sobre atividades físicas habituais, sendo considerado o tempo total em minutos e a frequência semanal. Este instrumento possui indicadores adequados de validade e alta concordância com a capacidade cardiorrespiratória em adolescentes. Os adolescentes foram classificados em insuficientemente ativos se realizassem menos de 300 minutos de atividades físicas semanais ${ }^{18}$.

Foram realizadas análises descritivas e inferenciais. Verificou-se a distribuição normal dos dados por meio do teste Kolmogorov Smirnov. Na estatística inferencial utilizou-se os testes U de Mann-Whitney e Kruskal-Wallis com post-hoc de Dunn para comparação das variáveis contínuas e para associação das variáveis categóricas, o teste Qui-Quadrado para heterogeneidade. As correlações foram analisadas por meio do teste de Spearman. Em função da variável dependente apresentar três categorias, recorreu-se à análise de regressão multinomial (categoria de referência: primeiro tercil do tempo sentado) para a análise de fatores associados. Foram incluídas no modelo ajustado apenas as variáveis que, na análise bruta, apresentaram $p<0,25^{19}$. Para todas as análises considerou-se um nível de significância de 5\%. Este projeto foi aprovado pelo Comitê de Ética envolvendo Seres Humanos da Universidade do Estado de Santa Catarina (Protocolo n. 535. 621). Aos menores de 18 anos foi entregue o Termo de Consentimento Livre e Esclarecido para assinatura dos pais ou responsáveis. E para todos os sujeitos da pesquisa foi entregue o Termo de Consentimento Livre e Esclarecido. Só participaram da pesquisa os sujeitos que assinaram o (s) referido (s) termo (s).

\section{RESULTADOS}

Foram avaliados 412 adolescentes da zona urbana e 104 da zona rural. Os dados descritivos da amostra estão apresentados na Tabela 1. Os adolescentes investigados apresentaram, em média, 14,57 (1,77) anos de idade. Embora a maior parte da amostra tenha uma renda média de 3,1 a 6 salários mínimos, verificou-se que 54,1\% dos adolescentes possuíam chefes de família com oito anos ou menos de estudo. Não foram identificadas diferenças na duração do sono entre os sexos $(p=0,642)$, tampouco na prevalência de baixa duração do sono $(p=0,218)$. As moças apresentaram maior prevalência de atividade física insuficiente $(p=0,003)$. Os adolescentes permaneceram, em média, 382,2 minutos sentados por dia, sendo este tempo superior no sexo feminino $(p=0,001)$ (Tabela 1).

O tempo sentado e a duração do sono, apesar de baixa magnitude, apresentaram correlação negativa ( $r=-0,198 ; p<0,001)$, sendo que quanto maior o tempo sentado, mais tarde eram seus horários de dormir $(r=0,134 ; p=0,002)$. Além disso, verificou-se diferença entre os grupos de adolescentes com oito ou mais horas de sono e menos de oito horas de sono com relação ao tempo sentado $(p=0,001)$ (Figura 1).

A pontuação média da PDSS foi de 15,1 pontos e observou-se, apesar da baixa magnitude, correlação positiva desta pontuação com a idade $(r=0,141 ; p=0,001)$, ou seja, quanto maior a idade dos adolescentes, maior a sonolência. Também foram observadas correlações significativas na duração do sono $(r=-0,107 ; p=0,015)$, tempo de atividade física $(r=-0,098 ; p=0,026)$ e tempo sentado $(p=0,229 ; p<0,001)$. Nos tercis da pontuação da PDSS, identificou-se maior tempo sentado nos valores superiores da escala $(p<0,001)$ e considerando apenas a primeira pergunta da PDSS relativa à sonolência em sala de aula (Figura 2).

Na Tabela 2 é apresentada a análise de associação entre o tempo sentado (moderado e alto) e as variáveis independentes. Em relação ao tempo sentado moderado, observou-se, na análise ajustada, que as variáveis sexo, faixa etária, turno de estudo, local de residência, renda e PDSS estiveram associadas ao desfecho. Essas associações revelaram que as
Tabela 1. Dados descritivos da amostra.

\begin{tabular}{|c|c|c|c|c|}
\hline Variáveis & Total & Rapazes & Moças & p-valor \\
\hline Idade, anos & $14,6(1,8)$ & $14,4(1,9)$ & $14,7(1,6)$ & 0,033 \\
\hline Faixas etárias, \% & & & & \multirow{5}{*}{0,041} \\
\hline $10-12$ & 15,3 & 18,6 & 11,9 & \\
\hline 13-14 & 27,3 & 29,7 & 24,9 & \\
\hline $15-16$ & 45,5 & 40,3 & 51,0 & \\
\hline $17-19$ & 11,8 & 11,4 & 12,3 & \\
\hline Local de domicílio, \% & & & & \multirow{3}{*}{0,662} \\
\hline Rural & 21,2 & 20,9 & 19,4 & \\
\hline Urbano & 79,8 & 79,1 & 80,6 & \\
\hline Turno de estudo, $\%$ & & & & \multirow{3}{*}{0,770} \\
\hline Noturno & 8,7 & 8,4 & 9,1 & \\
\hline Diurno & 91,3 & 91,6 & 90,9 & \\
\hline Renda familiar, $\%$ & & & & \multirow{4}{*}{0,642} \\
\hline$\leq 3$ salários mínimos & 20,0 & 23,2 & 16,9 & \\
\hline 3,1-6 salários mínimos & 50,2 & 45,6 & 54,9 & \\
\hline$>6$ salários mínimos & 29,8 & 31,2 & 28,5 & \\
\hline Escolaridade do chefe da família & & & & \multirow{3}{*}{0,024} \\
\hline$\leq 8$ anos & 54,1 & 58,9 & 49,0 & \\
\hline$>8$ anos & 45,9 & 41,1 & 51,0 & \\
\hline Duração do sono, h & $7,9(1,6)$ & $8,00(1,7)$ & $7,8(1,5)$ & 0,216 \\
\hline Baixa duração do sono, \% & 53,6 & 51,0 & 56,4 & 0,218 \\
\hline PDSS, pontos & $15,1(5,6)$ & $14,6(5,4)$ & $15,6(5,7)$ & 0,030 \\
\hline Sonolência na sala de aula & & & & \multirow{4}{*}{0,194} \\
\hline Nunca & 33,7 & 37,3 & 30 & \\
\hline Às vezes & 42,1 & 40,7 & 43,5 & \\
\hline Sempre & 24,2 & 22,1 & 26,5 & \\
\hline Percepção de estresse alto, $\%$ & 22,1 & 11,8 & 32,8 & $<0,001$ \\
\hline Atividade física semana, min & $\begin{array}{c}501,5 \\
(536,6) \\
\end{array}$ & $\begin{array}{c}562,9 \\
(523,9) \\
\end{array}$ & $\begin{array}{c}437,6 \\
(543,3) \\
\end{array}$ & $<0,001$ \\
\hline Insuficientemente ativos, $\%$ & 40,1 & 33,5 & 46,2 & 0,003 \\
\hline Tempo sentado, min & $\begin{array}{c}382,2 \\
(234,1) \\
\end{array}$ & $\begin{array}{c}352,1 \\
(225,8)\end{array}$ & $\begin{array}{c}413,6 \\
(238,9) \\
\end{array}$ & 0,001 \\
\hline
\end{tabular}

* p-valor do teste Qui-quadrado ou $\mathrm{U}$ de Mann-Whitney.

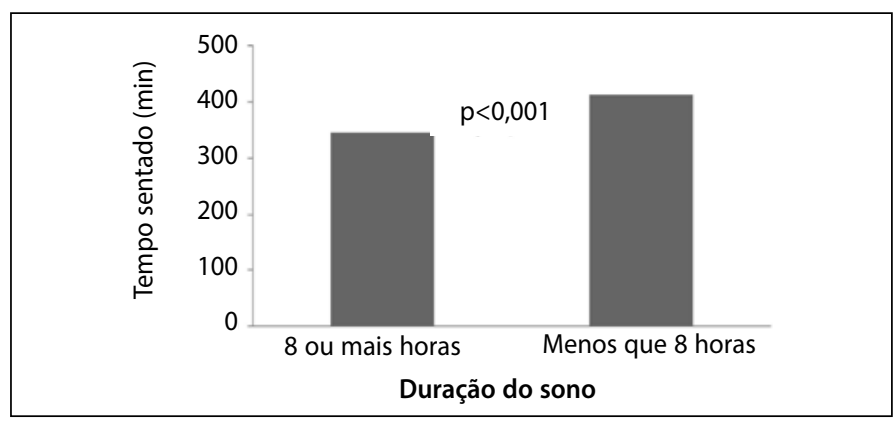

Figura 1. Tempo sentado de acordo com a duração do sono dos adolescentes.
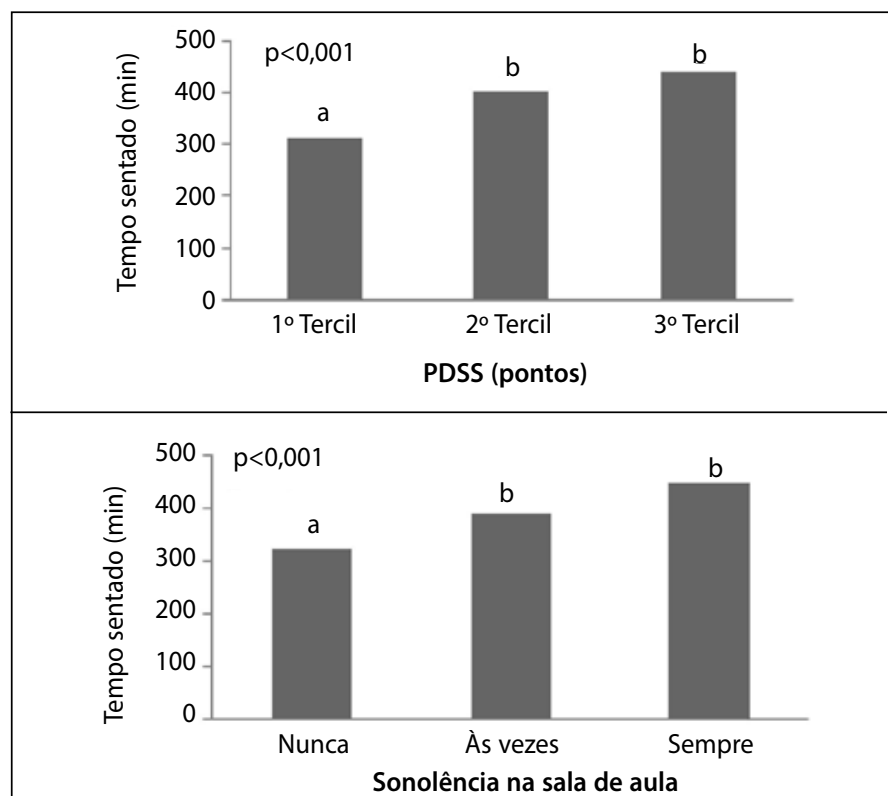

*p-valor do teste Kruskal-Wallis com post-hoc de Dunn, **letras diferentes remetem a médias estatisticamente diferentes.

Figura 2. Tempo sentado de acordo com as medidas de sonolência. 
Tabela 2. Análise de regressão multinomial considerando o tempo sentado como variável dependente.

\begin{tabular}{|c|c|c|c|c|c|c|c|c|}
\hline \multirow[b]{2}{*}{ Variáveis } & \multicolumn{4}{|c|}{ Tempo sentado moderado } & \multicolumn{4}{|c|}{ Tempo sentado alto } \\
\hline & $\begin{array}{c}\text { Prevalências } \\
(\%)\end{array}$ & $\begin{array}{c}\text { Bruta } \\
\text { OR (IC 95\%) } \\
\end{array}$ & $\begin{array}{c}\text { Ajustada } \\
\text { OR (IC 95\%) }\end{array}$ & p-valor* & $\begin{array}{c}\text { Prevalências } \\
(\%)\end{array}$ & $\begin{array}{c}\text { Bruta } \\
\text { OR (IC 95\%) } \\
\end{array}$ & $\begin{array}{c}\text { Ajustada } \\
\text { OR (IC 95\%) }\end{array}$ & p-valor* \\
\hline \multicolumn{8}{|l|}{ Sexo } & \multirow{3}{*}{0,016} \\
\hline Masculino & 22,8 & 1 & 1 & & 22,4 & 1 & 1 & \\
\hline Feminino & 32,0 & $2,05(1,34-3,12)$ & $2,01(1,25-3,24)$ & 0,004 & 30,4 & $1,98(1,29-3,03)$ & $1,88(1,12-3,13)$ & \\
\hline \multicolumn{8}{|l|}{ Faixa etária } & \multirow{5}{*}{$\begin{array}{c}0,002 \\
<0,001 \\
0,105\end{array}$} \\
\hline $17-19$ & 27,9 & $2,64(1,14-6,14)$ & $2,68(1,03-6,99)$ & 0,044 & 32,8 & $5,83(2,26-15,07)$ & $5,34(1,83-15,61)$ & \\
\hline $15-16$ & 32,3 & $3,64(1,90-6,98)$ & $3,18(1,56-6,50)$ & 0,001 & 34,5 & $7,27(3,25-16,23)$ & $5,97(2,51-14,21)$ & \\
\hline 13-14 & 23,4 & $1,52(0,76-3,06)$ & $1,52(0,72-3,20)$ & 0,268 & 19,1 & $2,33(0,99-5,51)$ & $2,12(0,85-5,28)$ & \\
\hline $10-12$ & 19,0 & 1 & 1 & & 10,1 & 1 & 1 & \\
\hline \multicolumn{8}{|l|}{ Turno de estudo } & \multirow{3}{*}{$<0,001$} \\
\hline Noturno & 24,4 & 1 & 1 & & 17,8 & 1 & 1 & \\
\hline Diurno & 27,6 & $1,44(0,69-3,01)$ & $3,01(1,31-6,94)$ & 0,009 & 27,2 & $1,95(0,86-4,44)$ & $6,06(2,28-16,12)$ & \\
\hline \multicolumn{8}{|l|}{ Local de Residência } & \multirow{3}{*}{0,020} \\
\hline Urbano & 28,9 & $1,89(1,10-3,25)$ & $1,96(1,08-3,58)$ & 0,026 & 28,2 & $2,03(1,16-3,54)$ & $2,18(1,13-4,20)$ & \\
\hline Rural & 21,2 & 1 & 1 & & 19,2 & 1 & 1 & \\
\hline \multicolumn{8}{|l|}{ Renda Familiar } & \multirow{4}{*}{$\begin{array}{l}0,001 \\
0,013\end{array}$} \\
\hline$\leq 3$ salários mínimos & 22,4 & 1 & 1 & & 19,3 & 1 & 1 & \\
\hline 3,1-6 salários mínimos & 29,9 & $2,30(1,40-3,79)$ & $2,16(1,24-3,78)$ & 0,007 & 36,4 & $3,25(1,98-5,33)$ & $2,56(1,44-4,58)$ & \\
\hline > 6 salários mínimos & 35,9 & $2,68(1,54-4,64)$ & $2,77(1,51-5,08)$ & 0,001 & 29,1 & $2,52(1,41-4,49)$ & $2,33(1,20-4,56)$ & \\
\hline \multicolumn{8}{|l|}{ Atividade física } & \multirow{3}{*}{0,012} \\
\hline Ativo & 26,4 & 1 & 1 & & 22,2 & 1 & 1 & \\
\hline Insuficientemente ativo & 28,8 & $1,46(0,95-2,24)$ & $1,26(0,79-2,01)$ & 0,329 & 32,7 & $1,97(1,28-3,03)$ & $1,89(1,15-3,09)$ & \\
\hline \multicolumn{8}{|l|}{ Grau de instrução } & \multirow{3}{*}{0,206} \\
\hline$\geq 8$ anos de estudo & 27,2 & 1 & 1 & & 21,9 & 1 & 1 & \\
\hline$<8$ anos de estudo & 27,4 & $1,25(0,82-1,90)$ & $0,91(0,56-1,49)$ & 0,721 & 31,6 & $1,80(1,17-2,75)$ & $1,40(0,84-2,34)$ & \\
\hline \multicolumn{8}{|l|}{ Percepção de estresse } & \multirow{3}{*}{0,207} \\
\hline Baixo estresse & 25,1 & 1 & 1 & & 25,9 & 1 & 1 & \\
\hline Alto estresse & 35,1 & $1,86(1,13-3,05)$ & $1,12(0,63-2,00)$ & 0,692 & 28,1 & $1,44(0,86-2,42)$ & $0,67(0,36-1,25)$ & \\
\hline \multicolumn{8}{|l|}{ Duração do sono } & \multirow{3}{*}{0,376} \\
\hline 8 ou mais horas & 25,3 & 1 & 1 & & 21,9 & 1 & 1 & \\
\hline$<8$ horas & 29,2 & $1,49(0,98-2,27)$ & $1,17(0,74-1,86)$ & 0,504 & 29,9 & $1,76(1,14-2,71)$ & $1,25(0,76-2,06)$ & \\
\hline \multicolumn{8}{|l|}{ PDSS** } & \multirow{4}{*}{$\begin{array}{l}<0,001 \\
<0,001\end{array}$} \\
\hline $1^{\circ}$ tercil & 26,4 & 1 & 1 & & 13,8 & 1 & 1 & \\
\hline $2^{\circ}$ tercil & 25,0 & $1,28(0,78-2,10)$ & $1,21(0,71-2,07)$ & 0,477 & 30,7 & $3,00(1,73-5,24)$ & $3,31(1,78-6,17)$ & \\
\hline $3^{\circ}$ tercil & 31,3 & $2,12(1,25-3,60)$ & $1,83(1,01-3,36)$ & 0,047 & 35,3 & $4,59(2,55-8,27)$ & $4,97(2,50-9,87)$ & \\
\hline
\end{tabular}

* p-valor da análise ajustada. ** Pediatric Day Time Sleepiness Scale.

moças apresentaram 1,88 (IC95\%: 1,12-3,13) vez mais chance de apresentar tempo sentado moderado em relação aos rapazes. Além disso, os adolescentes com faixa etária de 17-19 anos (OR=2,68; IC95\%=1,03-6,99) e de $15-16$ anos $(\mathrm{OR}=3,18 ; \mathrm{IC} 95 \%=1,56-6,50)$ apresentam maiores chances de dispender mais tempo sentado quando comparados aos mais novos.

Os adolescentes do turno diurno $(\mathrm{OR}=3,01 ; \mathrm{IC} 95 \%=1,31-6,94)$, residentes na região urbana $(\mathrm{OR}=1,96 ; \mathrm{IC} 95 \%=1,08-3,58)$, com renda de 3-6 salários $(O R=2,16$; IC95\%=1,24-3,78), superior a seis salários $(\mathrm{OR}=2,77 ; \mathrm{IC} 95 \%=1,51-5,08)$ e do 30 tercil da PDSS $(\mathrm{OR}=1,83 ; \mathrm{IC} 95 \%=$ $1,01-3,36)$ indicaram maiores chances de tempo sentado moderado.

Quanto àqueles que relataram permanecer muito tempo sentado, considerando as mesmas variáveis, foram identificadas associações. Adolescentes do sexo feminino $(\mathrm{OR}=1,88 ; \mathrm{IC} 95 \%=1,12-3,13)$, das faixas etárias de 17-19 (OR=5,34; IC95\%=1,83-15,61) e 15-16 anos $(O R=5,97$; $\mathrm{IC} 95 \%=2,51-14,21)$, do turno diurno $(\mathrm{OR}=6,06 ; \mathrm{IC} 95 \%=2,28-16,12)$, com renda familiar de 3-6 salários $(\mathrm{OR}=2,56$; IC95\%=1,44-4,58), superior a seis salários $(\mathrm{OR}=2,33$; IC95\%=1,20-4,56), e com níveis insuficientes de atividade física $(\mathrm{OR}=1,89 ; \mathrm{IC} 95 \%=1,15-3,09)$ expressaram maiores chances de ficar mais tempo sentado.

\section{DISCUSSÃO}

A prevalência dos distúrbios de sono na infância e adolescência é estimada entre $20 \%$ e $30 \% 20$. Os adolescentes investigados apresentaram prevalência de 53,6\% de baixa duração de sono, sendo superior aos 20\% identificados em adolescentes residentes em zonas rurais do Sul do Brasil' ${ }^{14}$ e aos 15,5\% identificados em adolescentes europeus ${ }^{21}$. Em relação à sonolência diurna, foi identificada média da PDSS de $15,09(5,6)$ pontos, sendo similar às médias encontradas na literatura15,22.

Correlações entre a duração do sono e a sonolência diurna foram investigadas em alguns estudos com adolescentes. Perez-Lloret et al. ${ }^{23}$., identificaram correlação embora significativa e negativa, de baixa magni- tude $(r=-0,36, p<0,01)$ e Moreno ${ }^{24}$ correlação significativa positiva entre as pontuações da PDSS e a quantidade de horas de sono $(p<0,001)$.

Na adolescência a baixa duração de sono está associada a aspectos importantes de saúde. Ela pode ter relação com maiores escores de tempo sentado associado aos comportamentos sedentários. Estes comportamentos estão relacionados ao elevado tempo de exposição à tela (televisão e videogames), apresentando implicações na saúde de adolescentes, como a supressão de produção de melatonina e redução da temperatura corporal noturna ${ }^{25}$.

O comportamento sedentário desencadeia a atenuação do ciclo de repouso-atividade, com efeitos adversos sobre o sono. Isto sugere que o declínio da atividade física no decorrer dos anos, pode prejudicar o sono ${ }^{26}$. No presente estudo, os adolescentes mais velhos apresentaram maiores chances de dispender maior tempo sentado e maior sonolência diurna do que os mais novos.

Existem outras ligações potenciais entre sono e comportamento sedentário. A diminuição das horas de sono eleva o período de tempo acordado, aumentando assim as oportunidades de movimento. No entanto, o sono insuficiente ou de má qualidade pode resultar em fadiga durante a vigília, com redução do gasto energético total. Com isto, baixos níveis de atividade física diária podem estar associados com a redução da quantidade de sono ${ }^{27}$. Logo, supõe-se que a baixa duração de sono e o aumento da sonolência diurna podem influenciar os adolescentes a optarem por ficar em frente às telas, a realizar atividades com maior dispêndio energético ${ }^{2}$. Isto ajuda a explicar as maiores chances apresentadas pelos adolescentes insuficientes ativos de Maravilha, SC, Brasil de dispender tempo sentado, quando comparados aos suficientementes ativos.

Associações entre o nível de atividade física e o comportamento sedentário tem sido objeto de investigação na área das ciências da saúde. Tais associações se sustentam em estudos como o de 
Katzmarzyk et al. ${ }^{28}$, em que apontaram a relação entre o maior tempo sentado e a mortalidade por inúmeras causas relacionadas a doenças cardiovasculares e câncer. De modo semelhante, Countryman et al. ${ }^{29}$., relataram em amostra de 367 adolescentes, associações entre baixo nível de atividade física e baixa duração do sono com o aumento do risco para síndrome metabólica e piora na aptidão cardiorrespiratória.

No presente estudo, 40,1\% dos adolescentes foram classificados como insuficientemente ativos. Este resultado é superior aos 25,4\% encontrados por Pelegrini e Petroski ${ }^{3}$ em escolares de Florianópolis-SC e inferior aos 58,2\% encontrados por Hallal et al. ${ }^{2}$ com adolescentes de Pelotas-RS. Embora a prevalência de adolescentes insuficientemente ativos de Maravilha, SC, Brasil tenha apresentado valores intermediário entre os dois estudos citados, ela pode ser considerada alta.

Não está claro na literatura qual o ponto de corte para o tempo sentado de modo a prevenir os riscos para a saúde. O tempo sentado observado em Maravilha, SC, Brasil foi ligeiramente inferior ao de adolescentes norte americanos (407 min) ${ }^{30}$. A ligeira diferença com a amostra de adolescentes norte-americanos, pode se relacionar as diferenças culturais, de costumes e hábitos entre os dois países.

Os pontos positivos destacados neste estudo foram: a) a promoção da discussão destas associações pouco investigadas na literatura; b) a utilização de uma amostra representativa; e c) por tratar-se de um município de pequeno porte, acredita-se que os resultados possam ser transformados em politicas de saúde pública com maior eficiência à população alvo. Apontam-se, como limitações do estudo a utilização de questionário como forma de avaliar a duração do sono de forma indireta. Além disso, algumas variáveis não foram contempladas neste estudo, como, por exemplo, a presença de distúrbios do sono como apneia e tempo de tela. Estas variáveis associadas ao desfecho, podem ser objetos de futuras investigações. Além disso, sugere-se que novas investigações descrevam, além da quantidade de tempo em atividade física, qual o horário que estas atividades acontecem considerando um possível efeito termorregulatório da atividade física no ciclo vigília-sono.

\section{CONCLUSÃO}

Mesmo em um município de pequeno porte, altas prevalências de duração do sono inadequado, de baixa atividade física e de alto tempo em comportamentos sedentários foram observadas. Adolescentes com menor duração do sono e maior sonolência diurna apresentaram maior tempo dedicado aos comportamentos sedentários. Assim, medidas de educação para um estilo de vida mais ativo na adolescência poderiam incluir atividades de instrução relacionadas à higiene do sono, considerando que este fator poderá ser fundamental na diminuição das atividades sedentárias e aumento das atividades físicas mais vigorosas. Sugerem-se, por fim, acompanhamentos longitudinais e experimentais para que se possa estabelecer, com maior clareza, as relações de causa e efeito entre o tempo sentado com o sono e a sonolência diurna nos adolescentes.

Todos os autores declararam não haver qualquer potencial conflito de interesses referente a este artigo.

CONTRIBUIÇÕES DOS AUTORES: Todos os autores contribuíram individualmente e de forma significante para a realização do manuscrito. EPGF (0000-0002-6924-122X)* RDA (0000-0003-0338-230X)*, DGB (0000-0002-5543-5445)* e CM (0000-0002-7317-738X)*, foram os principais contribuintes na redação do manuscrito. DF (0000-00027043-2015)* realizou a coleta de dados, organizou e tabulou os dados. AP (0000-0001-8862-9636)* e EPGF avaliaram os dados da análise estatística. EPGF, DF, DGB, RDA, CM, AP e TSB (0000-0003-2016-9287)*, realizaram a pesquisa bibliográfica, a revisão do manuscrito, discussão dos resultados e contribuíram com o conceito intelectual do estudo. Todos os autores contribuíram com o conceito intelectual e revisaram o artigo antes da submissão. *Número ORCID (Open Researcher and Contributor ID).

\section{REFERÊNCIAS}

1. Owen N, Sparling PB, Healy GN, Dunstan DW, Matthews CE. Sedentary behavior: emerging evidence for a new health risk. Mayo Clin Proc. 2010;85(12):1138-41.

2. Hallal $P C$, Bertoldi $A D$, Gonçalves $H$, Victora CG. Prevalence of sedentary lifestyle and associated factors in adolescents 10 to 12 years of age. Cad Saude Publica. 2006;22(6):1277-87.

3. Pelegrini A, Petroski EL. Inatividade física e sua associação com estado nutricional, insatisfação com a imagem corporal e comportamentos sedentários em adolescentes de escolas públicas. Rev Paul Pediatr. 2009;27(4):366-73.

4. Healy GN, Dunstan DW, Salmon J, Cerin E, Shaw JE, Zimmet PZ, et al. Breaks in sedentary time: beneficial associations with metabolic risk. Diabetes Care. 2008;31(4):661-6.

5. Spruyt K, Molfese DL, Gozal D. Sleep duration, sleep regularity, body weight, and metabolic homeostasis in school-aged children. Pediatrics. 2011;127(2):e345-52.

6. Liu X, Liu L, Owens JA, Kaplan DL. Sleep patterns and sleep problems among schoolchildren in the United States and China. Pediatrics. 2005;115(Suppl 1):241-9.

7. Ferreira LR, de Martino MM. Sleep patterns and fatigue of nursing students who work. Rev Esc Enferm USP. 2012;46(5):1178-83.

8. Pereira EF, Bernardo MP, D'Almeida V, Louzada FM. Sleep, work, and study: sleep duration in working and non-working students. Cad Saude Publica. 2011;27(5):975-84.

9. Andrade MM, Benedito-Silva AA, Domenice S, Arnhold IJ, Menna-Barreto L. Sleep characteristics of adolescents: a longitudinal study. J Adolesc Health. 1993;14(5):401-6.

10. Barger LK, Wright KP Jr, Hughes RJ, Czeisler CA. Daily exercise facilitates phase delays of circadian melatonin rhythm in very dim light. Am J Physiol Regul Integr Comp Physiol. 2004;286(6):R1077-84.

11. Brasil - Instituto Brasileiro de Geografia e Estatística [página na Internet]. Síntese de indicadores sociais 2010 [acessado em 10 de agosto de 2015]. Disponível em http://biblioteca.ibge.gov.br/ visualizacao/livros/liv45700.pdf

12. Raggio RL, Magnanini MMF. A lógica da determinação do tamanho da amostra em investigações epidemiológicas. Cad Saúde Col (Rio de Janeiro). 2000;8(2):9-28.

13. Pardini R, Matsudo S, Araújo T, Matsudo V, Andrade E, Braggion G, et al. Validação do questionário internacional de nível de atividade física (IPAQ-versão 6): estudo piloto em adultos jovens brasileiros. Rev Bras Ciên e Mov Brasília. 2001;9(3):39-44.

14. Pereira E, Teixeira CS, Louzada FM. Sonolência diurna excessiva em adolescentes: prevalência e fatores associados. Rev Paul Pediatr. 2010;28(1):98-103.

15. Drake C, Nickel C, Burduvali E, Roth T, Jefferson C, Pietro B. The pediatric daytime sleepiness scale (PDSS): sleep habits and school outcomes in middle-school children. Sleep. 2003;26(4):455-8.

16. Felden ÉP, Carniel JD, Andrade RD, Pelegrini A, Anacleto TS, Louzada FM. Translation and validation of the Pediatric Daytime Sleepiness Scale (PDSS) into Brazilian Portuguese. J Pediatr (Rio J). 2016;92(2):168-73.

17. Florindo AA, Romero A, Peres SV, Silva MV, Slater B. Development and validation of a physical activity assessment questionnaire for adolescents. Rev Saude Publica. 2006;40(5):802-9

18. Pate RR, Freedson PS, Sallis JF, Taylor WC, Sirard J, Trost SG, Dowda M. Compliance with physical activity guidelines: prevalence in a population of children and youth. Ann Epidemiol. 2002;12(5):303-8.

19. Hosmer DW, Lemeshow S. Applied logistic regression. 1989. New York: Johns Wiley \& Sons; 1989.

20. Hamilton GJ. Types and Treatment of Pediatric Sleep Disturbances. Psychology in the Schools. 2009;46(9):899-904

21. Carli V, Hoven CW, Wasserman C, Chiesa F, Guffanti G, Sarchiapone M, et al. A newly identified group of adolescents at "invisible" risk for psychopathology and suicidal behavior: findings from the SEYLE study. World Psychiatry. 2014;13(1):78-86.

22. Langberg JM, Dvorsky MR, Marshall S, Evans SW. Clinical implications of daytime sleepiness for the academic performance of middle school-aged adolescents with attention deficit hyperactivity disorder J Sleep Res. 2013;22(5):542-8.

23. Perez-Lloret S, Videla AJ, Richaudeau A, Vigo D, Rossi M, Cardinali DP, et al. A multi-step pathway connecting short sleep duration to daytime somnolence, reduced attention, and poor academic performance: an exploratory cross-sectional study in teenagers. J Clin Sleep Med. 2013;9(5):469-73.

24. Moreno T. Estudo da sonolência diurna e hábitos de sono numa população escolar dos 11-15 anos: validação em português da" Pediatric Daytime Sleepiness Scale" [dissertação]. Lisboa, Protugal: Universidade de Lisboa; 2013.

25. Higuchi S, Motohashi Y, Liu Y, Ahara M, Kaneko Y. Effects of VDT tasks with a bright display at night on melatonin, core temperature, heart rate, and sleepiness. J Appl Physiol (1985). 2003;94(5):1773-6.

26. Prinz PN. Age impairments in sleep, metabolic and immune functions. Exp Gerontol. 2004;39(11-12):1739-43

27. Tremblay MS, Esliger DW, Tremblay A, Colley R. Incidental movement, lifestyle-embedded activity and sleep: new frontiers in physical activity assessment. Can J Public Health. 2007;98(Suppl 2):208-17.

28. Katzmarzyk PT, Mason C. The physical activity transition. J Phys Act Health. 2009;6(3):269-80.

29. Countryman AJ, Saab PG, Llabre MM, Penedo FJ, McCalla JR, Schneiderman N. Cardiometabolic risk in adolescents: associations with physical activity, fitness, and sleep. Ann Behav Med. 2013;45(1):121-31

30. Sisson SB, Shay CM, Camhi SM, Short KR, Whited T. Sitting and cardiometabolic risk factors in U.S adolescents. J Allied Health. 2013;42(4):236-42. 